\title{
Medial Nucleus Accumbens Projections to the Ventral Tegmental Area Control Food Consumption
}

\author{
Colin W. Bond, ${ }^{1,2}{ }^{\circledR}$ Richard Trinko, ${ }^{1}$ Ethan Foscue, ${ }^{1}$ Kara Furman, ${ }^{1,2}{ }^{\circledR}$ Stephanie M. Groman, ${ }^{1}$ \\ Jane R. Taylor, ${ }^{1,3,4}$ and Ralph J. DiLeone $\mathrm{e}^{1,2,4,5}$ \\ ${ }^{1}$ Department of Psychiatry, Division of Molecular Psychiatry, Yale University School of Medicine, New Haven, Connecticut 06510, \\ ${ }^{2}$ Interdepartmental Neuroscience Program, Yale Graduate School of Arts and Sciences, New Haven, Connecticut 06510, ${ }^{3}$ Department of Psychology, \\ Yale University, New Haven, Connecticut 06510, ${ }^{4}$ Department of Neuroscience, Yale University, New Haven, Connecticut 06510, and ${ }^{5}$ Kavli \\ Institute for Neuroscience, Yale University, New Haven, Connecticut 06510
}

Decades of research have shown that the NAc is a critical region influencing addiction, mood, and food consumption through its effects on reinforcement learning, motivation, and hedonic experience. Pharmacological studies have demonstrated that inhibition of the NAc shell induces voracious feeding, leading to the hypothesis that the inhibitory projections that emerge from the NAc normally act to restrict feeding. While much of this work has focused on projections to the lateral hypothalamus, the role of NAc projections to the VTA in the control food intake has been largely unexplored. Using a retrograde viral labeling technique and real-time monitoring of neural activity with fiber photometry, we find that medial NAc shell projections to the VTA (mNAc $\rightarrow$ VTA) are inhibited during food-seeking and food consumption in male mice. We also demonstrate that this circuit bidirectionally controls feeding: optogenetic activation of NAc projections to the VTA inhibits food-seeking and food intake (in both sexes), while optogenetic inhibition of this circuit potentiates food-seeking behavior. Additionally, we show that activity of the NAc to VTA pathway is necessary for adaptive inhibition of food intake in response to external cues. These data provide new insight into NAc control over feeding in mice, and contribute to an emerging literature elucidating the role of inhibitory midbrain feedback within the mesolimbic circuit.

Key words: dopamine; feeding; mesolimbic; nucleus accumbens; reward; ventral tegmental area

\section{Significance Statement}

The medial NAc has long been known to control consummatory behavior, with particular focus on accumbens projections to the lateral hypothalamus. Conversely, NAc projections to the VTA have mainly been studied in the context of drug reward. We show that NAc projections to the VTA bidirectionally control food intake, consistent with a permissive role in feeding. Additionally, we show that this circuit is normally inactivated during consumption and food-seeking. Together, these findings elucidate how mesolimbic circuits control food consumption.

\section{Introduction}

Poor diet is the number one health risk factor in the United States (U.S. Burden of Disease Collaborators, 2018), contributing to nearly half of all cardiometabolic deaths (Micha et al., 2017). While half of overweight and obese people report dieting in the

\footnotetext{
Received Dec. 2, 2018; revised Apr. 15, 2020; accepted Apr. 22, 2020.

Author contributions: C.W.B., R.J.D., and K.F. designed research; C.W.B., E.F., and R.T. performed research; C.W.B., S.M.G., and R.J.D. analyzed data; C.W.B. wrote the first draft of the paper; C.W.B., S.M.G., R.T., J.R.T., and R.J.D. edited the paper; C.W.B. and R.J.D. wrote the paper; K.F. contributed unpublished reagents/analytic tools.

We thank Drs. Marina Picciotto, Alex Kwan, and Brendan Hare for valuable discussion on experimental design, statistical approaches, and confocal imaging assistance; and the State of Connecticut, Department of Mental Health and Addiction Services for support.

The authors declare no competing financial interests.

Correspondence should be addressed to Ralph J. DiLeone at ralph.dileone@yale.edu.

https://doi.org/10.1523/JNEUROSCI.3054-18.2020

Copyright $\odot 2020$ the authors
}

last year (Snook et al., 2017), few are successful, with an average of just $23 \%$ of initial weight loss maintained 5 years after a structured weight loss program (Anderson et al., 2001). Identification of the neural circuitry that controls feeding cessation is also critical for understanding eating disorders and developing effective interventions.

The NAc is a key region controlling the inhibition of food consumption. Electrophysiological investigations have demonstrated that approximately half of NAc shell neurons reduce firing rate before consumption (Taha and Fields, 2006; Krause et al., 2010). Additionally, pharmacological inactivation of the NAc with GABA receptor agonists (Stratford and Kelley, 1997; Basso and Kelley, 1999) and glutamate antagonists (MaldonadoIrizarry et al., 1995) results in dramatic increases in feeding. Conversely, electrical stimulation of the NAc and optogenetic activation of glutamatergic fibers within the NAc inhibit consumption (Krause et al., 2010; Prado et al., 2016). These data are 
consistent with a proposed "sensory sentinel" role for the NAc, whereby it allows for feeding during times of safety and triggers rapid cessation of feeding in response to a threat (Kelley et al., 2005). While previous research has focused on the NAc projections to the lateral hypothalamus $(\mathrm{LH})$ as a key downstream mediator (Maldonado-Irizarry et al., 1995; Stratford and Wirtshafter, 2012a; Urstadt et al., 2013; O'Connor et al., 2015), the contribution of other NAc targets to this feeding role remains less clear.

There is anatomic and functional evidence that NAc projections to the VTA may also mediate feeding behavior. The strongest response effects produced by NAc inactivation are observed in the rostromedial portion of the NAc shell (Reynolds and Berridge, 2001). The VTA is a direct target of this region of the NAc (Zahm and Heimer, 1993; Usuda et al., 1998; Yang et al., 2018), and $\mathrm{GABA}_{\mathrm{A}}$ and $\mu$-opioid agonist infusions into the NAc increase c-Fos activation in the VTA (Stratford and Kelley, 1999; Zhang and Kelley, 2000). Moreover, feeding induced by NAc inhibition is dependent on intact $\mathrm{GABA}_{\mathrm{A}}$ and $\mathrm{GABA}_{\mathrm{B}}$ signaling in the VTA (Miner et al., 2010), and NAc $\mu$-opioid agonist induced feeding is blocked by simultaneous inactivation of the VTA (Will et al., 2003). Recently, projections from medial NAc (mNAc) to the VTA has attracted renewed interest, particularly in mediating response to drugs of abuse (Bocklisch et al., 2013; Edwards et al., 2017; Gibson et al., 2018). However, the role of direct $\mathrm{mNAc} \rightarrow$ VTA projections in food intake and related behaviors remains unclear (Yang et al., 2018). We hypothesized that inhibitory NAc projections to the VTA play a role in permitting food intake.

\section{Materials and Methods}

\section{Animals}

Male and female Drd1a-Cre(+) mice and Drd1a-Cre(-) littermate controls (strain EY262, Gensat, back-crossed at least 10 generations to a C57BL/6J background, RRID:MMRRC_017264-UCD) weighing 20-30 g were used for photo-stimulation and Syb2-eGFP tracing studies. Female $\operatorname{Drd2-Cre(+)~mice~and~Drd2-Cre(-)~littermate~controls~(strain~ER44,~}$ Gensat, backcrossed at least 10 generations to a C57BL/6J background, RRID:MMRRC_032108-UCD) weighing 20-30 g were used for these studies for Syb2-eGFP tracing studies. Male C57BL/6J background mice (JAX:000664, RRID:IMSR_JAX:000664) weighing 20-30 g were used for fiber photometry and photo-inhibition studies. Mice that were reused across experiments are noted below in Materials and Methods. No sex differences were observed in these experiments. Consequently, we used exclusively male animals in the photo-inhibition and fiber photometry experiments.

All animals were group housed. Animals were on a $12 \mathrm{~h}$ light-dark cycle and provided standard chow and water ad libitum, except during behavioral training, and all animal procedures were performed in accordance with the protocol approved by the Yale Institutional Animal Care and Use Committee. During behavioral studies, animals were food restricted to $85 \%-90 \%$ body weight, unless otherwise specified. For food pellet training, animals consumed $20 \mathrm{mg}$ chow pellets (Bio-Serv F0163), $20 \mathrm{mg}$ sweetened chow pellets (Bio-Serv F0071), or $20 \mathrm{mg}$ high-fat (HF; 35\%) pellets (Bio-Serv F07162). For licking experiments, California Farms Organic Sweetened Condensed Milk was diluted to $10 \%$ by volume with tap water.

\section{Viral production and surgical procedures}

Viral production for AA2V-EF1a-DIO-Syb2-eGFP was accomplished using a triple-transfection, helper-free method and purified as described in detail previously (Hommel et al., 2003), using the flox-eGFP-synaptobrevin fusion construct (Land et al., 2014). AAV2-EF1a-DIO- $h C h R 2$ (H134R)-eYFP and AAV2-EF1a-DIO-eNPHR3.0-eYFP were purchased from the UNC Vector Core. AAV1-Syn-DIO-GCaMP6s-WPRE and
$A A V 6.2-C M V-P I-C r e-r B G$ were purchased from the Penn Vector Core. We purchased rgAAV2-pmSyn1-eBFP-Cre and AAV2-hSyn-DIO$m$ Cherry from Addgene.

Animals were anesthetized with $10 \%$ ketamine and $1 \%$ xylazine and placed in a stereotaxic frame (Stoelting). For photo-stimulation experiments, after craniotomy, Drdla-Cre(+) mice or Drdla-Cre- mice were bilaterally injected with $0.5 \mu \mathrm{l}$ of AAV2-EF1a-DIO-hChR2-eYFP into the mNAc (AP 1.2, ML - 0.6, DV -4.4) and a bilateral optical fiber cannula (200 $\mu \mathrm{m}$ core, $0.22 \mathrm{NA}$, Doric Lenses) was immediately placed into the VTA (AP -3.0, ML $-0.35, \mathrm{DV}-4.6$ ). For photo-inhibition experiments, WT mice were bilaterally injected with $0.5 \mu \mathrm{l}$ of either $A A V 2$ EF1a-DIO-eNPHR3.0-eYFP or AAV2-hSyn-DIO-mCherry in the mNAc and $0.3 \mu \mathrm{l}$ of $r g A A V 2-p m S y n 1-e B F P-C r e$ into the VTA, with a bilateral optical fiber immediately placed into the mNAc at the same location (200 $\mu \mathrm{m}$ core, $0.22 \mathrm{NA}$, Doric Lenses). For synaptic tracing experiments, $\operatorname{Drd1a}-\mathrm{Cre}(+)$ or $\mathrm{Drd2}-\mathrm{Cre}(+)$ animals receiving $0.5 \mu \mathrm{l} \mathrm{AAV2-EF1a-}$ $D I O-S y b 2-E G F P$ in the mNAc were not cannulated. For fiber photometry experiments, WT mice were unilaterally injected with $0.5 \mu \mathrm{l}$ of AAV1-Syn-DIO-GCaMP6s into the mNAc and $0.3 \mu \mathrm{l}$ of either rgAAV2pmSyn1-eBFP-Cre or AAV6.2-CMV-PI-Cre-rBG into the ipsilateral VTA, with unilateral optical fiber ( $400 \mu \mathrm{m}$ core, $0.48 \mathrm{NA}$, Doric Lenses) immediately placed into the mNAc at the same location. Each injection consisted of $\sim 10^{12}$ infectious particles per milliliter. Viral injections into the NAc were targeted to the medial shell, although some viral spread was detected in the medial core. Animals recovered for at least 2 weeks before behavioral testing.

The rgAAV2 and AAV6.2 serotypes were chosen for demonstrated high retrograde transduction efficiency (Aronoff et al., 2010; Löw et al., 2013; Salegio et al., 2013; Tervo et al., 2016). Based on histology, we observed qualitatively higher retrograde efficiency with the rgAAV2 serotype, but no difference in the photometry signal. Consequently, data from these groups were analyzed together.

\section{Apparatus}

For operant experiments, an operant chamber (Med Associates) was outfitted with a house light, two cue lights, and two nose ports (one active, one inactive) adjacent to the magazine. Nose port and magazine entry were monitored by IR beam breaks. For the lick distractor test, an operant chamber (Med Associates) was outfitted with a water bottle and electrical lick detection, two cue lights, and two auditory stimuli (tone and white noise). For fiber photometry experiments, chambers with either three ports on the opposite side from the magazine or two ports on the same side as the magazine were used. For conditioned place preference (CPP) experiments, a three-chamber apparatus (Med Associates), with distinguishable flooring and wall patterning in opposing chambers, was used. Behavioral activity was assessed using Med PC IV software for all experiments.

\section{Optogenetics}

Indwelling fiber optic cannulae were connected via dual fiber optic patch cord (200 $\mu \mathrm{m}$ core, $0.22 \mathrm{NA}$, Doric Lenses) to an intensity dividing rotary joint (FRJ_1x2_FC-2FC; Doric Lenses). The rotary joint was further connected via mono fiber optic patch cord $(200 \mu \mathrm{m}$ core, $0.22 \mathrm{NA}$, Doric Lenses) to a blue ( $473 \mathrm{~nm}$; OEM Laser Systems) or yellow laser $(593.5 \mathrm{~nm}$; OEM Laser Systems) located outside of the chamber. All photo-stimulation $(473 \mathrm{~nm})$ protocols were performed with a $10 \mathrm{~Hz}$, $15 \mathrm{~ms}$ pulse width protocol. All photo-inhibition protocols $(593.5 \mathrm{~nm})$ were performed with near constant light (1 min on, $1 \mathrm{~ms}$ off). Light transmission efficiency of fiber optic cannulae was measured before surgery. Based on the light transmission efficiency measured before surgery, laser power was adjusted before each animal session to achieve $\sim 5 \mathrm{~mW}$ power at the tip of each optic cannula.

\section{Free feeding}

Animals were tethered and placed in a polycarbonate cage without bedding, and two plastic weight boats were tapped to the cage in opposing corners. Animals could freely consume $20 \mathrm{mg}$ sweetened chow pellets from both weight boats for $30 \mathrm{~min}$. On choice experiments, animals could freely choose between $20 \mathrm{mg}$ HF pellets and $20 \mathrm{mg}$ chow pellets in 
the same apparatus. During training, animals were tethered to the fiber optic patch cord, and consumption over 3 min periods was monitored by experimenter for at least $3 \mathrm{~d}$ to habituate animals to the experimenter and chamber. On the test day, animals were noncontingently photostimulated in $3 \mathrm{~min}$ off/on epochs, and pellet consumption was recorded. Four of the male Cre(+) mice used for the HF versus chow choice test had been used previously for the chow free feeding experiment, separated by at least $2 \mathrm{~d}$ between stimulation.

\section{Operant behavior}

Animals were first given access to $20 \mathrm{mg}$ sweetened chow pellets in their home cage before testing. Animals were then trained to enter the magazine to retrieve a pellet. Each pellet was delivered $10 \mathrm{~s}$ after the prior pellet retrieval. Animals were trained for a minimum of $2 \mathrm{~d}$ and until $>30$ pellets were earned in a single session. After magazine training, animals were trained without optic fiber tethering on an FR1 task, in which each nose poke was rewarded with a pellet. A new trial did not begin until animals entered the magazine to retrieve the pellet. Retrieval was followed by a $5 \mathrm{~s}$ intertrial interval, after which the nose poke port was reactivated, indicated by a cue light. Training continued until $>40$ pellets were earned in a single $30 \mathrm{~min}$ session. Mice were then tethered to the optic fiber and trained for an additional $6 \mathrm{~d}$. To prevent delivered pellets from triggering a false-positive magazine entry, those entries that occurred within $300 \mathrm{~ms}$ of a nose poke were discarded. After training, animals were tested with either photo-stimulation or photo-inhibition in $3 \mathrm{~min}$ off/on epochs during a $30 \mathrm{~min}$ FR1 session. For the photo-stimulation experiment, 5 of the Cre $(+)$ males and 5 males and 2 females of the $\mathrm{Cre}(-)$ mice were used in the HF versus chow pellet test, separated by at least $2 \mathrm{~d}$ between stimulation.

\section{Progressive ratio}

After FR1 testing, a separate cohort of animals were tested using a progressive ratio schedule of reinforcement, in which each subsequent reward required exponentially more nose poke responses based on the formula $\left(5 \times \mathrm{e}^{0.2 \mathrm{n}}\right)-5$, rounded to the nearest integer, where $n=$ number of rewards earned (Richardson and Roberts, 1996). Each nose poke elicited $3 \mathrm{~s}$ of photo-stimulation. The session ended when $10 \mathrm{~min}$ had elapsed without the animal earning a reward.

\section{Food-induced CPP}

Animals were first allowed to habituate to a three-chamber apparatus for $15 \mathrm{~min}$. The following day, animals were tethered to the optical patch fiber placed into the middle chamber of the apparatus and allowed to explore all chambers for $15 \mathrm{~min}$ to provide an index of individual chamber preference. Animals were pseudo-randomly assigned for conditioning experiments to their preferred or nonpreferred chamber. Across $6 \mathrm{~d}$, animals were restricted to one chamber for $30 \mathrm{~min}$. On alternating days, food was placed in one chamber (e.g., paired chamber) and the other chamber contained no food (nonpaired side). After conditioning, animals were tethered and allowed to explore for $15 \mathrm{~min}$, with each entrance to the food-paired side inducing photo-stimulation. Time spent in each chamber was measured. All $\mathrm{Cre}(+)$ and $\mathrm{Cre}(-)$ mice used here had been used in the FR1 or progressive ratio operant experiments, separated by at least $2 \mathrm{~d}$ between stimulation.

\section{Lick distractor test}

Animals were food-deprived overnight before being placed into an operant chamber with $10 \%$ sweetened condensed milk in a water bottle equipped with an electrical lick detection system. Animals were maintained on mild food deprivation, 95\%-100\% body weight (average $99 \%$ body weight), for the rest of the experiment. Animals were allowed to freely lick for $30 \mathrm{~min}$ each day. Training continued for a minimum of $5 \mathrm{~d}$, and variation in total licking across 2 consecutive days was $<30 \%$. For testing, photo-inhibition was performed in $5 \mathrm{~min}$ epochs, for a total of three off and three on epochs. To account for greater variability in licking compared with food consumption behaviors, 5 min laser epochs were used. During light on epochs, lick initiation (three consecutive licks within $1.5 \mathrm{~s}$ ) triggered the presentation of a $500 \mathrm{~ms}$ auditory + visual distractor. To prevent habituation to the distractor cues, the combination of distractor cues was randomly chosen from two auditory stimuli (tone or white noise) and two visual stimuli (oppositely placed cue lights). All mCherry and Halo-expressing mice were used in both the FR1 inhibition operant experiment and the lick distractor test, separated by $\sim 7 \mathrm{~d}$ between experiments.

\section{Locomotor test}

Animals were tethered to the optic patch cord and placed in a polycarbonate cage without bedding, surrounded by an open field chamber with 16 infrared beams (Med Associates, ENV-515). Animals were allowed to habituate in the chamber for $30 \mathrm{~min}$ and then photo-stimulated in $3 \mathrm{~min}$ off/on epochs for $30 \mathrm{~min}$. Five males and 2 females of the $\operatorname{Cre}(+)$ mice and 6 males of the $\operatorname{Cre}(-)$ mice were used in the operant FR1 experiment, separated by at least $\sim 2 \mathrm{~d}$ between experiments.

\section{Photometry}

Mice with indwelling $2.5 \mathrm{~mm}$ fiber optic ferrule were briefly anesthetized and connected to a fiber optic patch cord ( $400 \mu \mathrm{m}$ core, $0.48 \mathrm{NA}$; Doric Lenses), held in place by a zirconia sleeve. Animals were allowed to recover and move freely. Ratiometric fiber photometry was performed as previously described (Lerner et al., 2015), with each LED set to $40 \mu \mathrm{W}$ power at the end of the patch cord. These signals were then combined by a longpass dichroic mirror (DMLP425; Thorlabs) and collimated into the fiber optic patch cable.

Using custom script written in MATLAB software, each signal was low pass filtered with a cutoff frequency of $2 \mathrm{~Hz}$. The $490 \mathrm{~nm}$ excitation signal was regressed against the $405 \mathrm{~nm}$ control signal, and $\Delta \mathrm{F} / \mathrm{F}$ was calculated using the $490 \mathrm{~nm}$ residuals ( $490 \mathrm{~nm}$ residuals $/ 490 \mathrm{~nm}$ predicted). Behavioral events from the operant chamber (rewarded pokes, nonrewarded nose pokes, and magazine entries) were recorded via TTL signals generated by Med-PC IV (Med Associates). Nonrewarded nose pokes after a correct nose poke and nonrewarded nose pokes during the intertrial interval were algorithmically categorized as perseverative and premature, respectively. The $\Delta \mathrm{F} / \mathrm{F}$ signal from each trial was normalized by a $z$ score generated from the $-5 \mathrm{~s}$ to $-2 \mathrm{~s}$ before the behavioral event. Area under the curve (AUC) was calculated by integrating the $2 \mathrm{~s}$ before a behavioral event, the $2 \mathrm{~s}$ after a behavioral event, and the subsequent $2 \mathrm{~s}(2-4 \mathrm{~s}$ after event).

Video recordings of each session were collected, and the time of pellet consumption was visually confirmed to correspond with a magazine entry. Additionally, mice often made consecutive magazine entry beam breaks while staying in the magazine to search for and retrieve food pellets. To algorithmically categorize consecutive magazine entries, magazine entries followed by a consumption event, nose poke, or $>5 \mathrm{~s}$ until the next consecutive magazine entry were considered the end of a magazine bout. This allowed us to separate magazine entries into consummatory and nonconsummatory bouts of magazine activity.

\section{Histology}

Immunohistochemistry was performed on all mice that underwent viral surgery and experimentation. The sample sizes provided in the results for each experiment reflect the number of animals used for immunohistochemistry. Animals were deeply anesthetized and intracardially perfused with $4 \%$ PFA. The brain was removed and postfixed in PFA; and after immersion in sucrose, $40 \mu \mathrm{m}$ sections were made on a freezing microtome and stored in $1 \times$ PBS with $0.01 \%$ sodium azide. Immunohistochemistry was performed according to methods described previously (Narayanan et al., 2012). Staining for TH (mouse anti-TH; Millipore MAB318, RRID:AB_2201528; 1:10,000), GFP (chicken antiGFP; Abcam ab13970, RRID:AB_300798; 1:1000), or Cre (mouse antiCre; Millipore MAB 3120, RRID:AB_2085748; 1:1000) with secondary antibodies (Alexa-405, Alexa-488, Alexa-555; Abcam 175658 RRID:AB_ 2687445, Jackson ImmunoResearch Laboratories 703-545-155 RRID: AB_2340375, Thermo Fisher Scientific A31570 RRID:AB_2536180) was performed in $3 \%$ normal donkey serum and $0.3 \%$ Triton X-100. Tissue was visualized and images were captured using a fluorescent microscope (Carl Zeiss) using standard DAPI, FITC, and TRITC filter cubes. 


\section{Statistical analyses}

No statistical methods were used to predetermine sample sizes, but our sample sizes are similar to those reported in previous publications. Cage littermates were assigned to experimental or control groups on the basis of genotype and were age- and weight-matched. Comparisons were made between $\mathrm{Drdla}-\mathrm{Cre}(+)$ and $\mathrm{Drd1a}$ $\mathrm{Cre}(-)$ animals, and between mCherry and Halo-expressing animals, using a two-way ANOVA (with light, genotype, time, and chamber as factors). An unpaired two-tailed $t$ test was used for analysis of progressive ratio responding, and a Kolmogorov-Smirnov test on the pooled frequency distribution (by genotype) of nose pokes during progressive ratio responding.

To test the hypothesis that magazine entries and nose pokes were correlated with a significant change in fiber photometry fluorescence, we used a bootstrap method in which $10 \mathrm{~s}$ of fluorescence was randomly sampled from each animal's session, with replacement, proportional to the number of trials each animal performed. The random samples were $z$-scored as described above, and the mean was calculated. We performed 1000 iterations, allowing us to generate 99\% CIs for changes in fluorescence during a $10 \mathrm{~s}$ period.

To analyze dynamic changes in fluorescence and compare behavioral trials types, AUCs were analyzed using a linear mixed-effects model in $\mathrm{R}$ ( $\mathrm{R}$ project; http://www.r-project.org) to account for the imbalance in trial number between mice and imbalance in trial types within mice. This imbalance in the number of observations prevented the use of ANOVA for data analysis. Linear mixed-effects models, however, are able to account for datasets consisting of different numbers of observations, such as that reported here. In this linear mixed-effects model, epoch and outcome type were included as repeated, fixed effects and subject included as a random effect. Post hoc tests of significant interactions consisted of computing low-order comparisons between outcome type (consumption vs no consumption).

Threshold for significance was set at $p<0.05$ for all tests of significance. GraphPad Prism 7.0 (RRID:SCR_002798) was used for analysis for all behavioral data and R statistical package (RRID:SCR_001905) along with custom-written MATLAB 2017b software (RRID:SCR 001622) for photometry data. Code is available on request. Error bars indicate SEM.

\section{Results}

\section{Medial D1 accumbens neurons project throughout the} rostrocaudal extent of the ventral VTA

Circuit tracing studies using retrograde chemical tracers have characterized the NAc projections to the VTA as solely expressing dopamine D1 receptors (Bocklisch et al., 2013; Edwards et al., 2017; Yang et al., 2018) or containing a minority D2 projection (Lu et al., 1998; Gibson et al., 2018). To determine the pattern of D1- and D2-expressing NAc projections within the VTA, we used a genetically defined anterograde viral tracing strategy (Fig. 1A). AAV2-EF1a-DIO-Syb2-EGFP was injected into the mNAc of D1rd1a-Cre mice (D1-Cre, $n=7$, all female), leading to robust expression in the mNAc shell (Fig. $1 B$ ). The use of the Syb2-eGFP fusion protein enabled identification of NAc synaptic innervation within the VTA (Land et al., 2014). The bulk of synapses from mNAc were observed in the medioventral VTA (adjacent to intrafascicular nucleus and paranigral area) throughout the rostrocaudal extent of the of the VTA (Fig. 1C,D). Putative
VTA dopaminergic (VTA-DA) neurons were identified by immunohistochemical labeling of $\mathrm{TH}^{+}$cells. In the rostral VTA, NAc synapses were largely ventral to VTA-DA neurons (Fig. $1 C)$. In the medial and caudal VTA, synaptic contacts were observed adjacent to VTA-DA neurons (Fig. 1D,H). Minimal Syb2-eGFP expression was detected in the VTA of Drd2a-Cre animals (Fig. 1I,J), consistent with less innervation from D2expressing neurons into the VTA. The images selected for D2 projections to the VTA represent the stronger examples of the observed staining.

\section{Photo-stimulation of mNAc $\rightarrow$ VTA inhibits food consumption}

We next sought to determine the functional role of this pathway in food consumption. We injected AAV2-EF1a-DIO-hChR2eYFP into the mNAc of D1-Cre(+) or D1-Cre(-) littermates and implanted an optical fiber in the VTA to stimulate VTA-projecting terminals (Fig. 2A). Food-deprived animals were tethered and allowed to freely consume sweetened chow pellets from plastic dishes on either side of a polycarbonate cage (Fig. 2D). During training, consumption was monitored for at least $3 \mathrm{~d}$ to allow the animals to habituate and generate a stable baseline ( $<25 \%$ variation from $3 \mathrm{~d}$ average). We found that photo-stimulation of the mNAc $\rightarrow$ VTA pathway inhibited food consumption in the $\mathrm{Cre}(+)$ group during laser-on epochs (Fig. $2 E ; 2 \times$ repeated-measures ANOVA, genotype [Cre-, Cre +$] \times$ time, $F_{(9,126)}=5.07, p=7.57 \times 10^{-6}, n=7$ [4 males and 3 females] for Cre(-) and $n=9$ [7 males and 2 females] for $\operatorname{Cre}(+)$; Bonferroni-corrected post hoc analysis showed significant difference between genotypes during laser-on epochs, ${ }^{\star} p<0.05$ ). Total consumption was reduced in $\mathrm{Cre}(+)$ but not $\mathrm{Cre}(-)$ animals (Fig. 2F; $2 \times$ repeated-measures ANOVA, genotype [Cre-, Cre+ $] \times$ light $[$ on, off $], F_{(1,14)}=18.7, p=6.98 \times 10^{-4}$; Bonferroni-corrected post hoc analysis showed significant difference of laser epoch of the Cre $(+)$ group, $\left.{ }^{* * *} p<0.0001\right)$. 
A

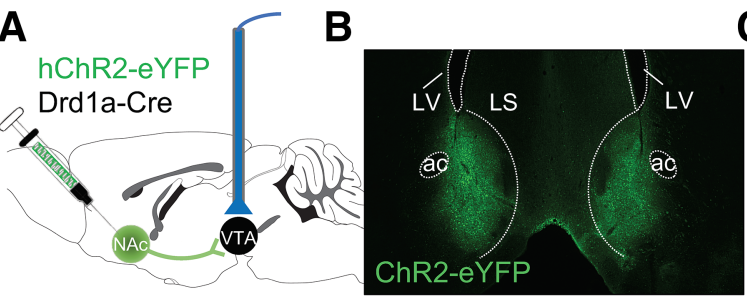

D

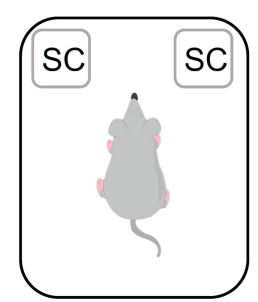

$E$

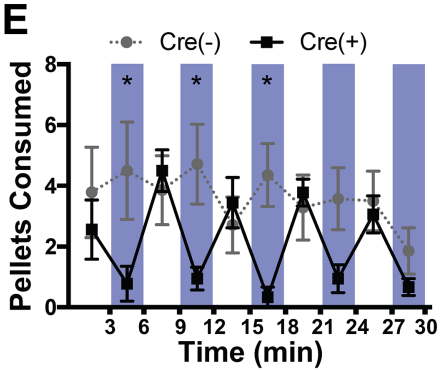

G

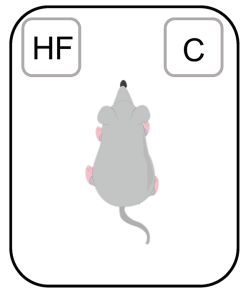

$\mathbf{H}$

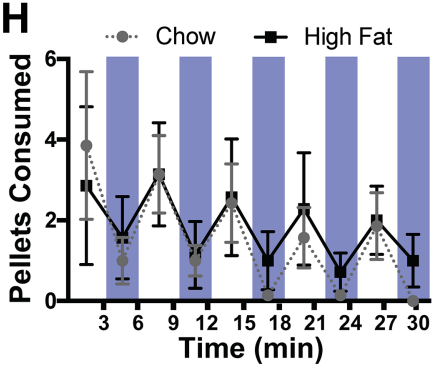

C

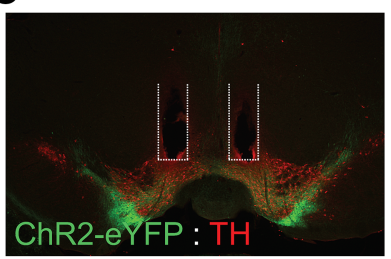

$\mathbf{F}$
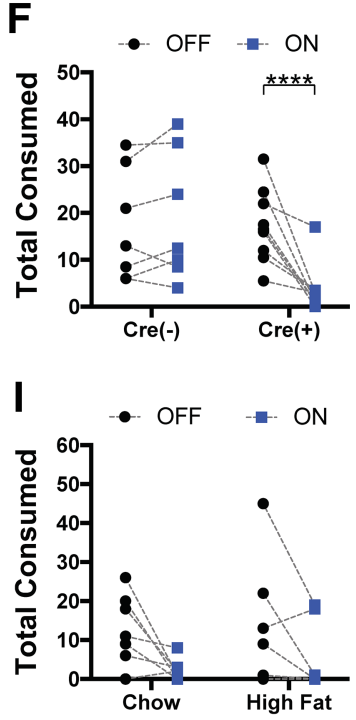

Figure 2. Activation of $m N A c \rightarrow V T A$ pathway inhibits consumption. $A$, Schematic showing injection of AAV2-EF1a-DIOhChR2-eYFP into the NAc shell and medial core of D1-Cre mice. B, Fluorescence image showing ChR2-eGFP expression (green, $488 \mathrm{~nm}$ ) in the NAc of D1-Cre animals. C, Fluorescence image showing ChR2-eGFP expression (green, $488 \mathrm{~nm}$ ) in the VTA, identified by TH (red, $555 \mathrm{~nm}$ ). Optical fiber placement adjacent to medial VTA (white dashed lines). D, Schematic of consumption experiment. Animals were allowed free access to plastic trays containing $20 \mathrm{mg}$ sweetened chow (SC) pellets for $30 \mathrm{~min}$. $\boldsymbol{E}$, Summary data showing decreased consumption in Drd1a- $\mathrm{Cre}(-)$ versus Drd1a-Cre(+) animals during laser-on epochs (blue shading). Mice were stimulated at $10 \mathrm{~Hz}$ ( $15 \mathrm{~ms}$ pulse width, $3 \mathrm{~min}$ on, $3 \mathrm{~min}$ off, repeating). $\boldsymbol{F}$, Total consumption summated across laser-on and laser-off epochs, as well as genotype. $\mathbf{G}$, Schematic of the HF versus chow pellet choice experiment. Animals were allowed free access to plastic trays containing $20 \mathrm{mg} H F$ pellets and chow pellets $(C)$ for $30 \mathrm{~min}$. $\boldsymbol{H}$, Summary data showing decreased consumption of both HF and ( pellets in Drd1a-Cre(+) animals during laser-on epochs, with no difference in food. $I$, Total consumption summated across epochs. All summary data are mean \pm SEM. ${ }^{*}$, ${ }^{* * *}$ Significant post hoc test.

Previous research has implicated the mNAc $\rightarrow$ VTA pathway in the control of HF consumption (Will et al., 2003). To determine whether activation of the $\mathrm{mNAc} \rightarrow \mathrm{VTA}$ circuit preferentially affects macronutrient intake, we injected $A A V 2-E F 1 a-D I O-$ $h C h R 2-e Y F P$ into the mNAc of D1-Cre $(+)$ mice. Animals were trained to freely choose between chow pellets and HF pellets (Fig. 2G). We found a reduction in consumption of both regular chow and HF pellets, but no difference in food preference (Fig. $2 \mathrm{H} ; 2 \times$ repeated-measures ANOVA, condition [chow, HF] $\times$ time; $F_{(9,54)}=0.187, p=0.995$; main effect of time, $F_{(9,54)}=6.917$, $p=1.49 \times 10^{-6}, n=7$ [ 5 males and 2 females]). Total chow pellet and total HF pellet consumption were both reduced following stimulation (Fig. 2 I; $2 \times$ repeated-measures ANOVA, condition [chow, HF] $\times$ light [on, off], $F_{(1,6)}=0.168, p=0.696$; main effect of laser epoch, $\left.F_{(1,6)}=32.8, p=0.001\right)$.

\section{Photo-stimulation of $\mathrm{mNAc} \rightarrow$ VTA inhibits operant responding for food without affecting motivation or locomotion}

The exact mechanism by which the accumbens mediates intake is unclear. One possibility is that the NAc efferents exert control over the motor circuits underlying consummatory actions (Kelley et al., 2005; Baldo and Kelley, 2007). To determine whether activation of the $\mathrm{mNAc} \rightarrow \mathrm{VTA}$ circuit inhibited consumption through direct inhibition of consummatory motor actions, animals were trained to operantly respond for sweetened chow pellets on an FR1 schedule and then tested with photo-stimulation during the FR1 task. We injected AAV2-EF1a-DIOhChR2-eYFP into the mNAc of D1-Cre $(-)$ and D1-Cre $(+)$ and found that activation significantly reduced operant responding during the session (Fig. 3A; $2 \times$ repeated-measures ANOVA, genotype [Cre-, Cre +$] \times$ time, $F_{(9,162)}=2.60$, $p=0.0079 ; n=13$ [10 males and 3 females] for $\mathrm{Cre}(+)$; and $n=7$ [5 males and 2 females] for Cre(-); Bonferroni-corrected post hoc analysis showed significant difference between genotypes during first laser epoch, $\left.{ }^{* *} p<0.001,{ }^{*} p<0.05\right)$. The reduction in pellets consumed toward the end of the session (in both stimulated and nonstimulated) is most likely due to a satiety effect. Summated operant responding for food across the entire session was significantly different (Fig. $3 B ; 2 \times$ repeatedmeasures ANOVA, genotype [Cre-, Cre+] $\times$ light [on, off], $F_{(1,18)}=4.73$, $p=0.0431$; Bonferroni-corrected post hoc analysis showed significant difference in laser epoch of the $\mathrm{Cre}(+)$ group, $\left.{ }^{* *} p<0.01\right)$. These results demonstrate that $\mathrm{mNAc} \rightarrow \mathrm{VTA}$ circuit activation suppresses both food-seeking and food consumption, and provides additional evidence that NAc feeding effects are not solely due to impairment of consummatory motor functions.

We next sought to determine whether activation of the mNAc $\rightarrow$ VTA circuit reduced the motivation to consume, thereby decreasing intake. To test this, animals trained on the FR1 schedule were then tested on a progressive ratio schedule, in which each pellet requires a greater number of nose pokes. Photo-stimulation was triggered for $3 \mathrm{~s}$ after every nose poke. We found a reduction in response rate, resulting in longer times to reach break point for Cre $(+)$ animals (Fig. $3 C$; Cre $(-)$, Cre $(+)$, Kolmogorov-Smirnov test, $D=0.127, p=5.04 \times 10^{-16}$; $n=4$ [2 males and 2 females] for Cre $(-)$; and $n=5$ [ 4 males and 1 female] for Cre $(+))$. However, activation of the mNAc $\rightarrow$ VTA circuit had no effect on the number of pellets earned, a measure of motivation (Fig. $3 D$; unpaired $t$ test, $p=0.643, t=0.484$ ).

One potential concern is that activation of the $\mathrm{mNAc} \rightarrow \mathrm{VTA}$ pathway inhibits locomotion, leading to a general reduction in behavior and possibly the slower responding rate observed under the progressive ratio schedule. Animals were tested in an open field chamber, with laser noncontingently activated in $3 \mathrm{~min}$ epochs after $30 \mathrm{~min}$ of habituation. We found a nonstatistically significant effect on locomotion, when analyzing laser-on/laseroff epochs (Fig. $3 E ; 2 \times$ repeated-measures ANOVA, genotype $\left[\right.$ Cre-, Cre+] $\times$ time, $F_{(9,189)}=1.91, p=0.052$, with the first 
30 min excluded from statistical analysis; $n=9$ [ 6 males and 3 females] for Cre(-); and $n=14$ [10 males and 4 females] for Cre $(+))$. Total locomotion during laser epochs was not affected (Fig. 3F; $2 \times$ repeated-measures ANOVA, genotype [Cre-, Cre+] $\times$ light [on, off], $F_{(1,21)}=$ 2.73, $p=0.114$ ).

To determine whether activation of this circuit instead altered the reinforcing effects of food, animals were trained to associate one chamber of a threechamber apparatus with food receipt. Photo-stimulation of the $\mathrm{mNAc} \rightarrow$ VTA circuit did not alter this conditioning (Fig. $4 A, B ; 2 \times$ repeated-measures ANOVA, genotype [Cre-, Cre+] $\times$ chamber [unpaired, middle, paired], $F_{(2,34)}=0.477, p=0.625 ; n=6$ [5 males and 1 female] for Cre(-); and $n=13$ [10 males and 3 females] for $\mathrm{Cre}(+))$. Together, these data indicate that activation of the mNAc $\rightarrow$ VTA circuit is not sufficient to produce CPP or aversion.

\section{Photo-inhibition of $\mathrm{mNAc} \rightarrow$ VTA} potentiates consumption

Previous research has shown that optogenetic inhibition of D1 neurons in the NAc is sufficient to increase consumption and protects against adaptive inhibition of food consumption in response to external cues (O'Connor et al., 2015), consistent with a the proposed "sensory sentinel" role of the NAc in feeding (Kelley et al., 2005). We sought to determine whether the $\mathrm{mNAc} \rightarrow$ VTA circuit mediates this sensory sentinel function.

We used a retrograde viral labeling approach to specifically label midbrainprojecting neurons from the mNAc in C57BL/6J male mice. Briefly, we used the $\mathrm{rgAAV}$ serotype, engineered for high-efficiency retrograde transport (Tervo et al., 2016), to deliver recombinase to the VTA. Either AAV2-EF1a-DIOeNPHR3.0-eYFP or control virus was injected into the mNAc, and an optical fiber placed over it, allowing for photo-inhibition of only midbrain projecting NAc neurons (Fig. $5 A, B$ ).

To determine whether inactivation of the $\mathrm{mNAc} \rightarrow \mathrm{VTA}$ pathway potentiates food-seeking, animals were first trained to perform an operant food-seeking task (FR1), as described above. After training, animals were returned to an ad libitum home cage diet for $2 \mathrm{~d}$, allowing them to return to full body weight. They were then tested with $3 \mathrm{~min}$ epochs of photo-inhibition during operant food-seeking. The number of pellets animals earned across $3 \mathrm{~min}$ trial blocks was not differentially altered by photo-stimulation compared with mCherry controls (Fig. 5C; $\times$ repeated-measures ANOVA, condition [mCherry, Halo] $\times$ time, $F_{(9,180)}=1.558, p=0.131 ; n=11$ [all males]; with a significant effect of time $\left(F_{(9,20)}=4.416, p=0.049\right)$. However, we found a significant increase in total food intake during photo-inhibition epochs, compared with control animals (Fig. $5 D ; 2 \times$ repeatedmeasures ANOVA, condition [mCherry, Halo] $\times$ light [on, off],
$F_{(1,20)}=5.50, p=0.0295$; Bonferroni-corrected post hoc analysis show significant difference in laser epoch only within the Halo group, $\left.{ }^{\star} p<0.05\right)$.

We next examined the role of the $\mathrm{mNAc} \rightarrow \mathrm{VTA}$ circuit in adaptive inhibition of food-seeking. To do this, we used the stimulus distractor behavioral paradigm, previously described (O'Connor et al., 2015). Briefly, to allow real-time monitoring of intake, animals were trained to lick for a palatable solution (10\% sweetened condensed milk). Lick burst initiation, defined as a minimum of three licks within $1.5 \mathrm{~s}$, was monitored in real time. During 5 min alternating epochs, yellow laser was delivered, and each lick burst initiation triggered a $500 \mathrm{~ms}$ auditory and visual stimulus. In control animals, the distractor stimulus reduced intake during the distractor/laser-on epochs, while photo-inhibition was protective against sensory distraction in the Halo group (Fig. $5 E ; 2 \times$ repeated-measures ANOVA, condition [mCherry, Halo] $\times$ time, $F_{(5,90)}=2.82, p=0.0206 ; n=11$ : all male, for mCherry and $n=9$ : all male, for Halo; Bonferroni-corrected post hoc analysis shows significant difference between viral treatments during the second laser/distractor epoch, $p<0.05$ ). Cumulative licks were not differentially altered in Halo-infected mice 

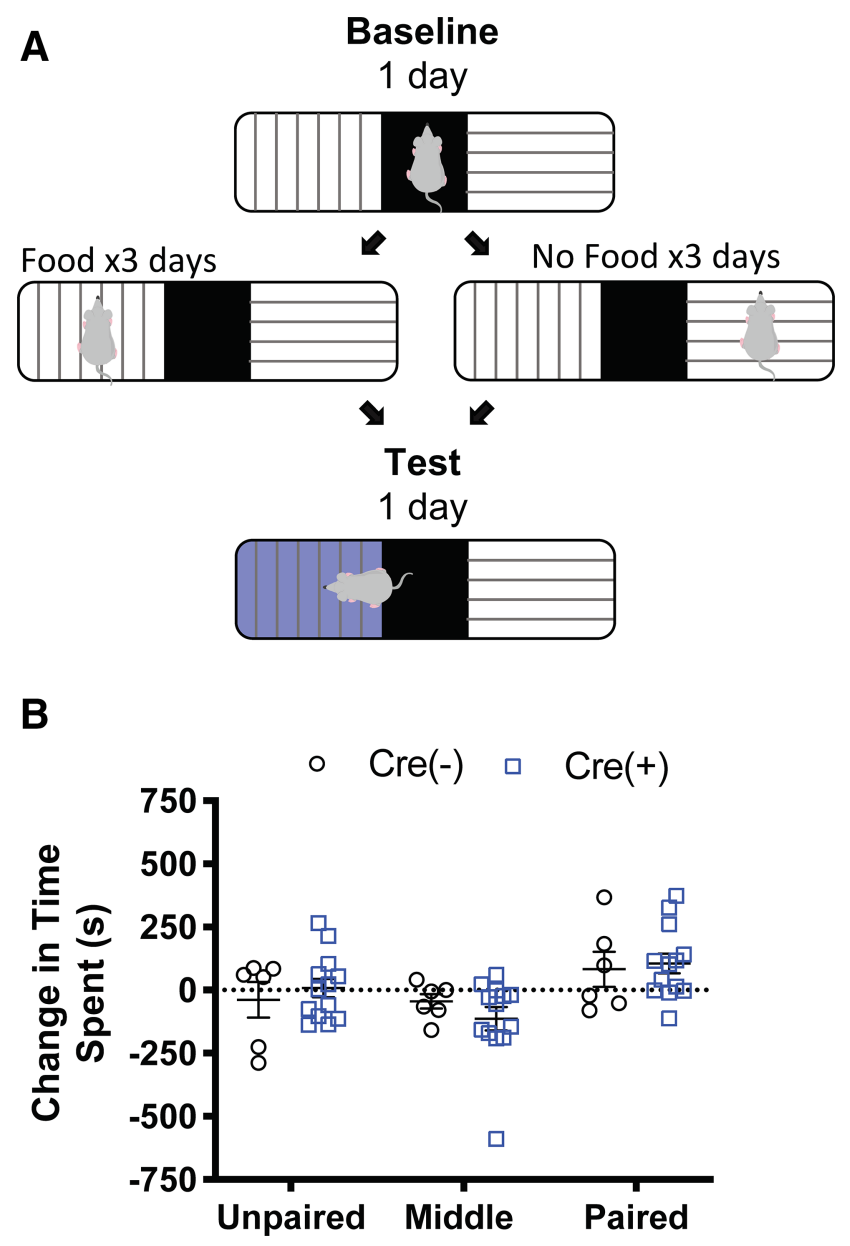

Figure 4. Activation of mNAc $\rightarrow$ VTA does not affect the valence associated with food. $\boldsymbol{A}$, Schematic of three-chamber food CPP experiment. Conditioning order (laser paired or tethered) was counterbalanced. After conditioning with food, animals were tested against baseline preference with photo-stimulation $(10 \mathrm{~Hz}, 15 \mathrm{~ms}$ pulse width) on entry into the foodpaired chamber. $\boldsymbol{B}$, Summary data showing absolute change in time spent (test - baseline) in each chamber (unpaired, middle, paired).

(Fig. 5F; $2 \times$ repeated-measures ANOVA, condition [mCherry, Halo] $\times$ light [on, off], $F_{(1,18)}=2.89, p=0.107$; main effect of viral treatment, $\left.F_{(1,18)}=7.05, p=0.0161\right)$. These findings support the hypothesis that the mNAc acts as a sentinel, adaptively inhibiting food consumption in response to external cues, and that NAc projections to VTA contribute to this function.

\section{mNAc $\rightarrow$ VTA dynamics during food-seeking and consumption}

Previous research has demonstrated reductions in firing rates in a subset of NAc neurons during feeding and food-seeking (Roitman et al., 2005; Taha and Fields, 2005, 2006; Krause et al., 2010). To determine whether the mNAc $\rightarrow$ VTA pathway demonstrates this activity pattern, we characterized the neural dynamics of mNAc $\rightarrow$ VTA neurons during food-seeking and consumption. Cre-recombinase with high retrograde activity was virally delivered into the VTA (rgAAV2-pmSyn1-eBFP-Cre), and AAV1-Syn-DIO-GCaMP6s-WPRE into the NAc, selectively labeling this pathway in C57BL/6J mice (Fig. 6A). This retrograde viral strategy led to robust GCaMP6s labeling in the NAc (Fig. 6B). An optical fiber was placed over the NAc, allowing us to monitor activity via fiber photometry, as previously described (Lerner et al., 2015). Animals were trained to perform an operant task on an FR1 schedule, allowing us to separately examine activity of this circuit in food-seeking, approach, and consumption behaviors.

During operant responding, we observed a decrease in fluorescence after a nose poke response (Fig. $6 C ; n=600$ nonreinforced trials, 471 reinforced trials, from 10 male WT mice). Importantly, this inhibition occurred on both reinforced and nonreinforced operant responses, indicating that the change in fluorescent signal was not due to food presentation or associated cues (Fig. 6E). We also observed a ramping in activity during approach to the magazine. On trials in which the animal retrieved and consumed a food pellet, but not on trials without consumption, we observed a delayed decrease in fluorescence after a magazine entry (Fig. $6 D ; n=191$ entry trials, 348 consumption trials). The black line (Fig. 6D,E) indicates average fluorescence signal that was significantly different from baseline with a bootstrap-generated 99\% CI. A significant reduction in fluorescence was observed during the $2-4$ s postentry period during a consumption trial versus entry only (Fig. $6 F ; F_{(2,1602)}=$ 4.17, $p=0.0157$ for summary data; post hoc $\chi^{2}$ test of independence, $p=0.000605$, Holm method-corrected).

To determine whether this decrease was related to the onset of consumption, we analyzed a subset of trials in which the mice took extended time to successfully retrieve the pellet (as assessed by multiple magazine entry beam breaks for a single reward), thus temporally separating magazine approach and entry from eventual pellet retrieval and consumption. These trials were broken down into the initial magazine entry, represented by the first beam break (with or without subsequent consumption), and magazine exit events, represented by the final beam break (with or without consumption). Magazine exits revealed neuronal inhibition on consummatory trials (Fig. $7 A, C ; F_{(2,792)}=3.53$, $p=0.0298$ for summary data; post hoc $\chi^{2}$ test of independence, $p=0.00734$, Holm method-corrected). Fluorescence following magazine entries with a single beam break were similar to the pooled population, showing increased fluorescence followed by inhibition on trials in which the animal consumed (Fig. $7 B, D$; $F_{(2,795)}=3.23, p=0.0402$ for summary data; post hoc $\chi^{2}$ test of independence, $p=0.0113$, Holm method-corrected). Together, this analysis indicates a suppression of activity in the $\mathrm{mNAc} \rightarrow$ VTA circuit during operant responding for food and consumption with an increase in activity likely occurring during magazine approach/entry and pellet retrieval. This pattern of inhibition is consistent with a permissive role for the $\mathrm{mNAc} \rightarrow \mathrm{VTA}$ circuit in mediating food-seeking with more complex dynamics during retrieval and consumption.

\section{Discussion}

In this study, we examined the role of mNAc projections to the VTA in controlling food intake. Consistent with previous reports, we found that D1 MSNs are the primary neuronal subtype projecting to VTA (Bocklisch et al., 2013; Edwards et al., 2017; Yang et al., 2018), and that the mNAc largely innervates the ventromedial VTA (Zahm and Heimer, 1993; Usuda et al., 1998; Yang et al., 2018). While we did observe minimal D2 neuronal projections in several mice, the majority of animals evaluated showed little to no evidence of D2 projections, suggesting a predominant D1 projection to the region. Our study builds on previous literature showing that the NAc controls food intake by demonstrating that the mNAc $\rightarrow$ VTA projection bidirectionally regulates feeding. Furthermore, we found that the mNAc $\rightarrow$ VTA circuit is necessary for distractor stimuli to suppress food consumption. Finally, we showed that the activity in this circuit is 
inhibited during food-seeking and food consumption. Together, these findings indicate that inhibition of the mNAc $\rightarrow$ VTA circuit is necessary to permit feeding.

It has been proposed that the NAc restricts intake via control over the motor behaviors that comprise a feeding response (Kelley et al., 2005). We find that the mNAc $\rightarrow$ VTA circuitry bidirectionally regulates instrumental actions, not solely food consumption actions, consistent with previous reports that pharmacological inhibition of the NAc increases operant responding for food (Pulman et al., 2012; Stratford and Wirtshafter, 2012b). However, we did not find a statistically significant effect on locomotion or activity alone. The pattern of activity in the Cre $(+)$ animals during open field locomotion was suggestive of a change, and the nonsignificant trend toward decreased locomotion during laser-off epochs is consistent with a recent report (Yang et al., 2018). Observation during free feeding experiments did not reveal any obvious changes in locomotor or stereotypic activity. Importantly, this potential locomotor effect during laser-off epochs is not sufficient to explain the significant decreases in feeding and food-seeking we observe during laser-on epochs.

Given the well-established role of the VTA in reward-related learning and reinforcement, it is possible that activation of inhibitory mNAc projections generates an aversive or anhedonic state. Surprisingly, we found little evidence that activation of this circuit impacts the motivational or rewarding properties of food consumption as measured by food preference, the progressive ratio task, and food CPP. These findings are consistent with a recent report that selective activation of the $\mathrm{mNAc} \rightarrow \mathrm{VTA}$ pathway does not induce real-time place preference (Yang et al., 2018) and pharmacological evidence that NAc inhibition with GABA agonists does not affect progressive ratio responding (Zhang et al., 2003).

Importantly, we found that $\mathrm{mNAc} \rightarrow \mathrm{VTA}$ circuit activity is inhibited during food-seeking and food consumption, consistent with a restrictive role in consumption. While others have suggested that the NAc acts to gate consumption (Krause et al., 2010), the temporal dynamics of fiber photometry using GCaMP6s do not allow us to determine whether circuit inhibition occurred before seeking and consumption. Interestingly, we also observed a ramping in activity during approach to the food magazine, similar to the reported ramping in the dorsal striatum during approach (London et al., 2018). This phenomenon, activity ramping before inhibition during a food consumption task, has also been observed with electrophysiological recordings in the NAc (Roitman et al., 2005; Krause et al., 2010). Notably, the ramping we observe does not appear to depend on food consumption or detection of food per se, suggesting a broader role in approach behavior. To better understand this ramping activity
B

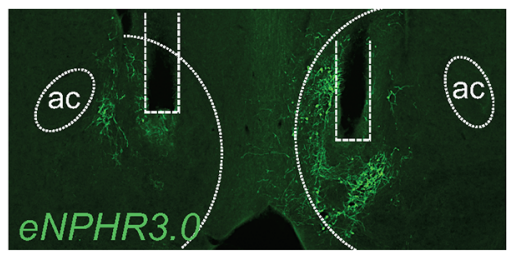

C
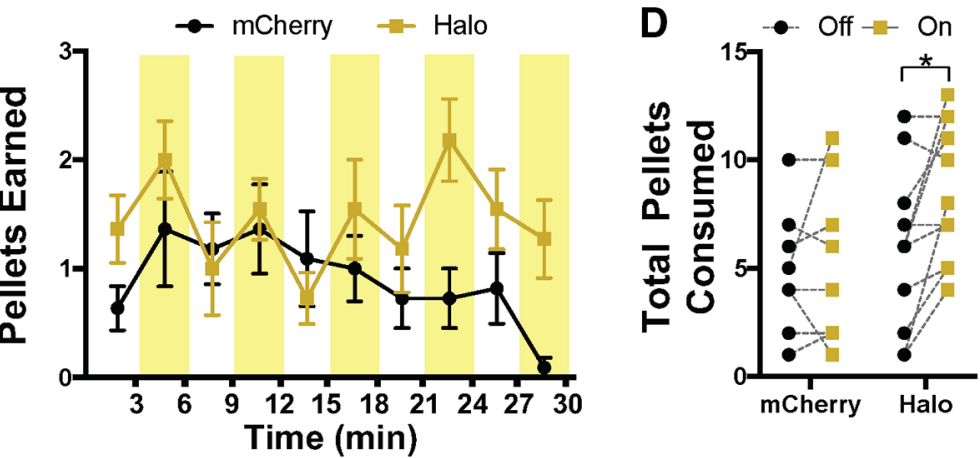

$\mathbf{F}$

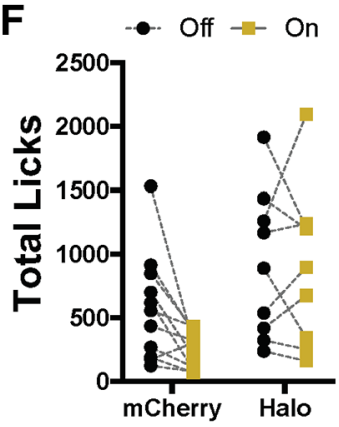

Figure 5. Inactivation of $\mathrm{mNAC} \rightarrow \mathrm{VTA}$ pathway promotes consumption. $\boldsymbol{A}$, Schematic showing injection of $0.5 \mu \mathrm{l}$ represents laser-on and distractor-on epochs. $\boldsymbol{F}$, Total licks summated across laser-on and laser-off. All summary data are mean \pm SEM. *Significant post hoc test.

of mNAc neurons, further investigation with finely timed manipulations of the NAc during approach specific behaviors is needed.

Given the similar feeding effects reported during optogenetic activation of $\mathrm{mNAc} \rightarrow \mathrm{LH}$ projections (O'Connor et al., 2015), antidromic activity and activation of $\mathrm{LH}$ projecting collaterals are a potential confound for the results reported here. Additionally, if there is high collateralization to the $\mathrm{LH}$ and VTA, the effects we observed during NAc photo-inhibition may be mediated by LH projections. However, dual-retrograde tracing shows little evidence of such collateralization between these regions in addition to evidence of functional segregation (Gibson et al., 2018). Supporting this, we found qualitatively less eNPHR3.0-eYFP labeling in the LH compared with the VTA with our projection-specific retrograde viral labeling of $\mathrm{mNAc} \rightarrow$ VTA circuit, indicating a lack of collateralization.

The results presented here potentially influence the interpretation of the observed decrease in intake during optogenetic 
A GCaMP6s Retrograde Cre

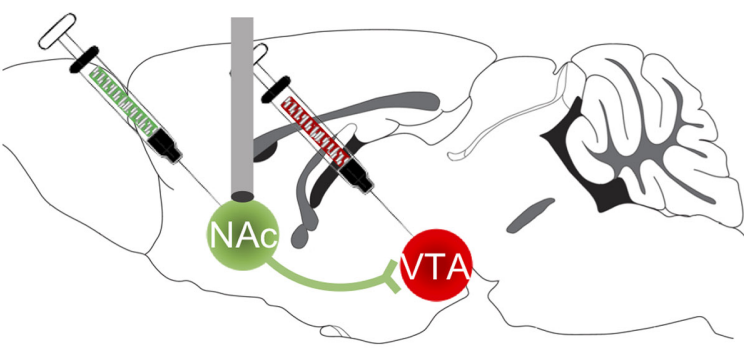

C

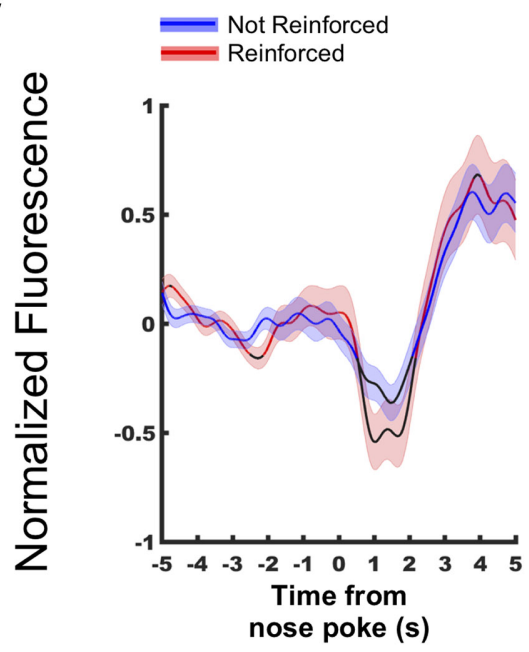

$\mathbf{E}$

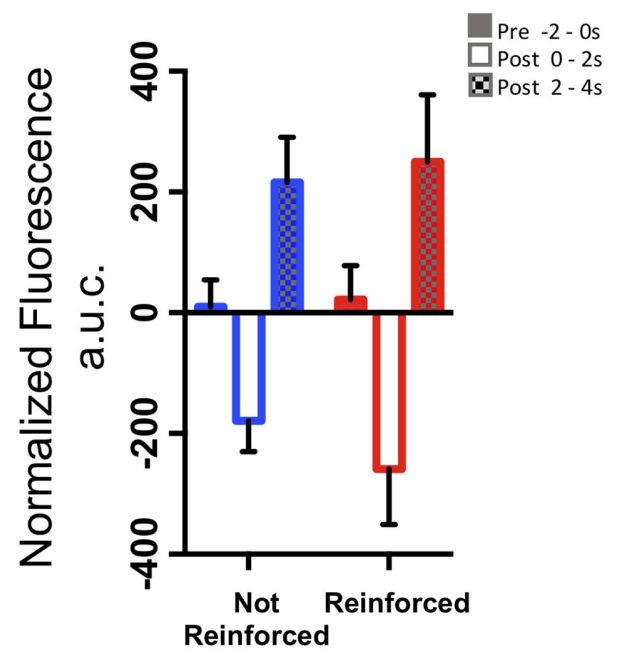

B GCaMP6s

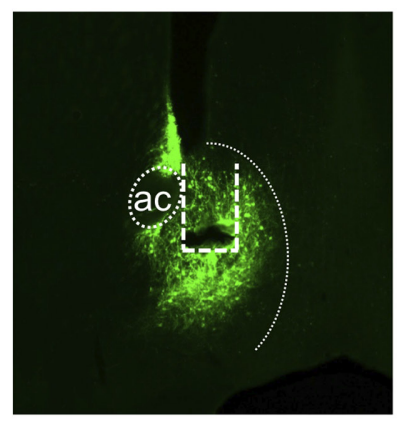

D

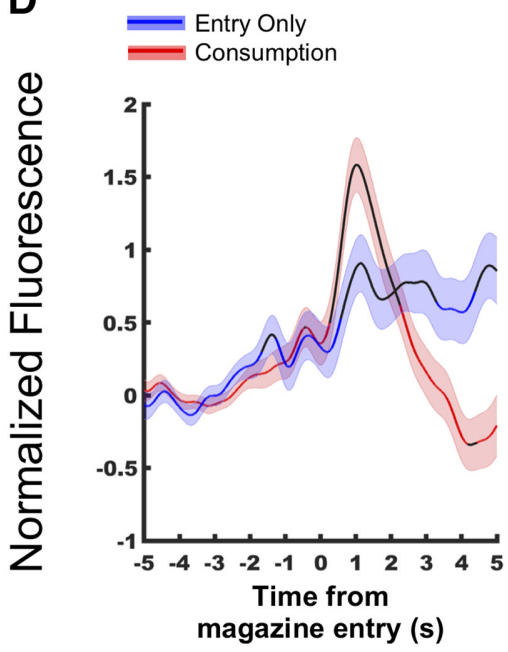

$\mathbf{F}$

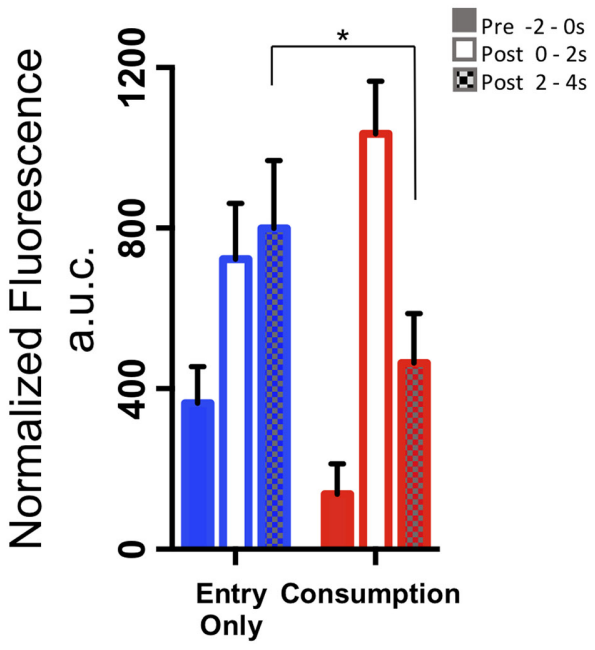

Figure 6. $\quad \mathrm{mNAC} \rightarrow$ VTA pathway is dynamically activated during reward seeking. $\boldsymbol{A}$, Schematic showing viral injection strategy; $0.5 \mu \mathrm{I}$ AAV1-Syn-GCaMP6s was delivered into the NAc shell, and $0.3 \mu \mathrm{l}$ retrograde Cre (rgAAV2-pmSyn1-eBFP-Cre or AAV6.2-CMV-PI-Cre-rBG) into the VTA, selectively labeling VTA-projecting neurons in the accumbens. $\boldsymbol{B}$, Fluorescence image showing GCaMP6s expression (green, $488 \mathrm{~nm}$ ) in the NAc. Optical fiber scarring evident adjacent to NAc (white dashed lines). C, Summary data showing normalized fluorescence during nonreinforced and reinforced nose pokes. Line indicates average signal. Shading represents SEM for all trials. Black center line indicates average fluorescence signal significantly different from baseline (bootstrap-generated 99\% CI). D, Summary data showing normalized fluorescence during magazine entries with and without consumption. Line indicates average signal. Shading represents SEM for all trials. Black center line indicates average fluorescence signal significantly different from baseline (bootstrap-generated $99 \%$ Cl). $\boldsymbol{E}$, Summary data of AUC for -2 to $0 \mathrm{~s}$ before nose poke, $0-2$ $\mathrm{s}$ after, and 2-4 s. $\boldsymbol{F}$, Summary data of AUC for -2 to $0 \mathrm{~s}$ before entry, $0-2 \mathrm{~s}$ after, and 2-4 $\mathrm{s}$ after showing an increase in signal on approach to the magazine, followed by a significant during consumption trials. Significant interaction between trial type and AUC epoch. All summary data are mean \pm SEM. *Post hoc $\chi^{2}$ test of independence $(p=0.000605)$.

stimulation of D1 terminals in the LH (O'Connor et al., 2015), as VTA-projecting D1 collaterals pass through the medial forebrain bundle adjacent to the LH. Terminal activation in the LH may also stimulate these VTA-projecting fibers. Further research with specific, projection- defined labeling, or double-dissociation strategies would be required to distinguish the relative contributions of the NAc projections to the LH and VTA in feeding behavior as both LH and VTA target sites mediate behavioral responses. 
A
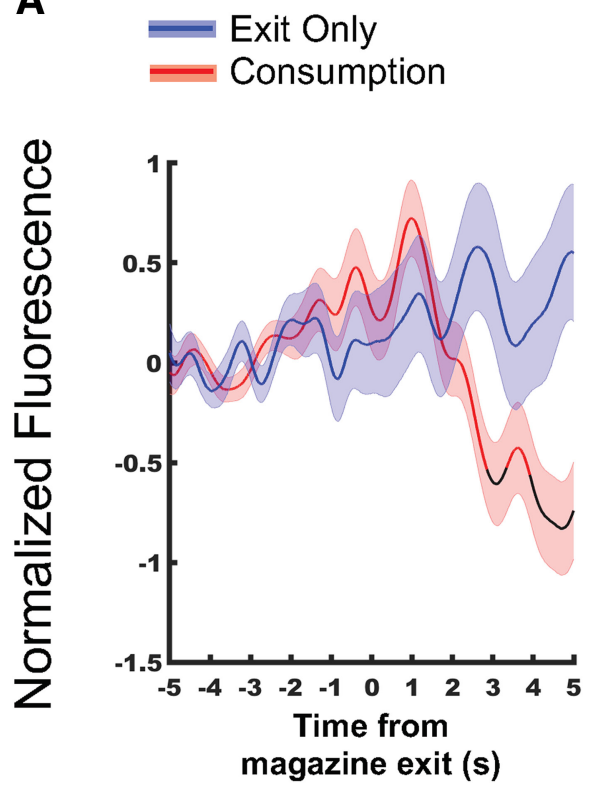

C

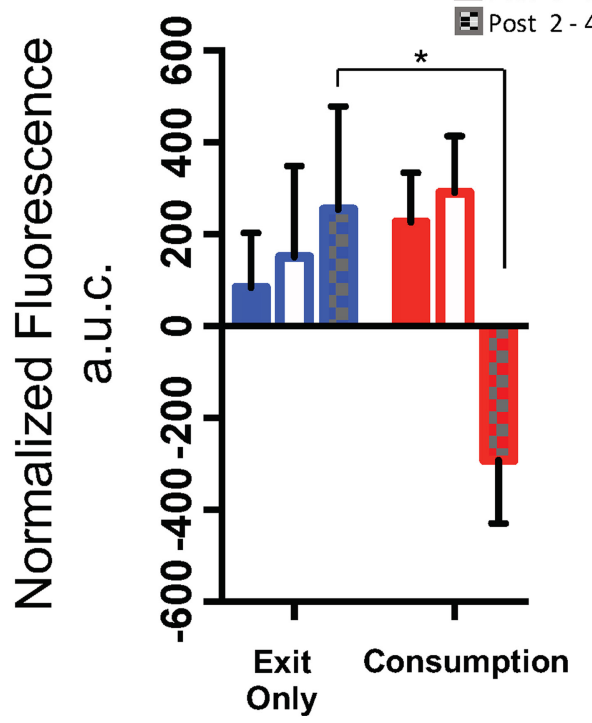

B
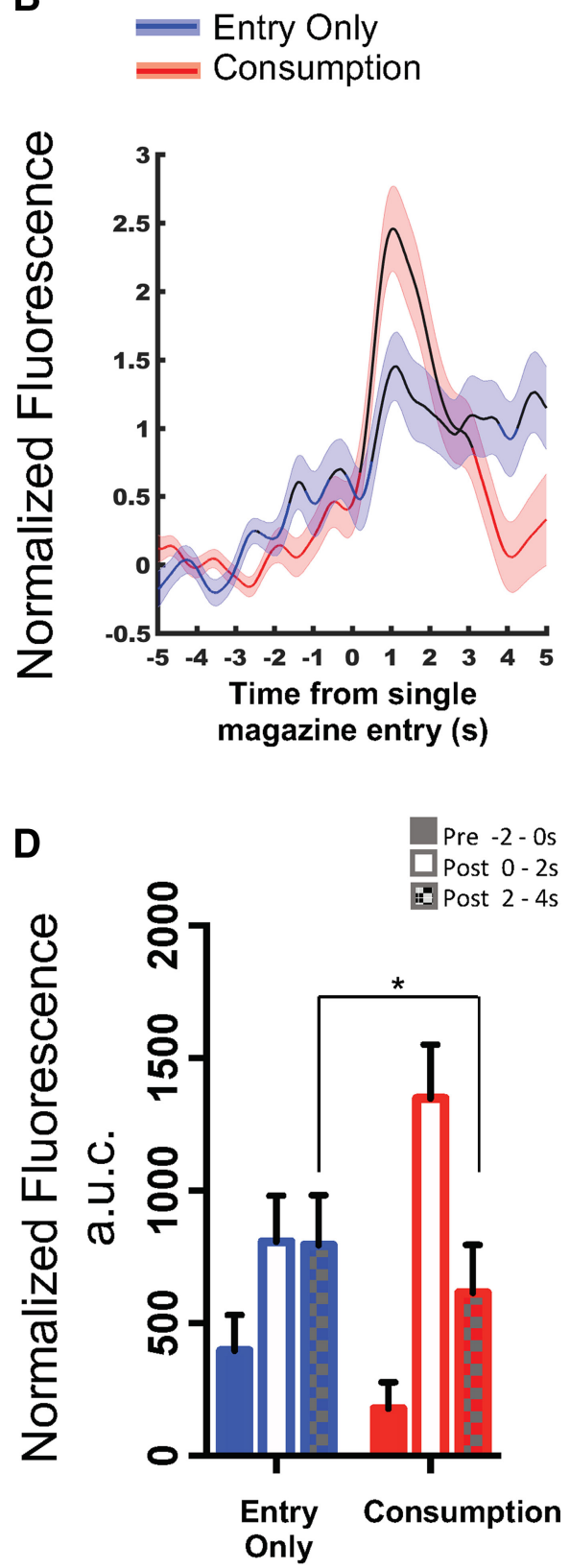

Figure 7. Dynamic inhibition of $\mathrm{mNAc} \longrightarrow \mathrm{VTA}$ fluorescence is related to consumption. $\boldsymbol{A}$, Summary data showing normalized fluorescence during magazine exits with and without consumption. Line indicates average signal. Shading represents \pm SEM for all trials. Black center line indicates average fluorescence signal significantly different from baseline (bootstrap-generated $99 \%$ (I). B , Summary data showing normalized fluorescence during single-beam break magazine entries with and without consumption. Black center line indicates average fluorescence signal significantly different from baseline (bootstrap-generated $99 \%$ Cl). C, Summary data of AUC for -2 to 0 s before exit, $0-2 \mathrm{~s}$ after, and 2-4 s after showing a significant decrease in fluorescence during consumption trials. Significant interaction between trial type and AUC epoch. *Post hoc $\chi^{2}$ test of independence $(p=0.00734)$. D, Summary data of AUC for -2 to 0 s before exit, $0-2$ $s$ after, and 2-4 s after showing a significant decrease in fluorescence during consumption trials. Significant interaction between trial type and AUC epoch. ${ }^{*}$ Post hoc $\chi^{2}$ test of independence $(p=0.0113)$. All summary data are mean \pm SEM.

The direct downstream neurons in the VTA mediating these feeding effects remain unclear. While previous electrophysiological investigations found little evidence for direct synaptic innervation of VTA DA neurons by the accumbens (Xia et al., 2011; Bocklisch et al., 2013), viral tracing studies identified robust connections to VTA DA neurons (Watabe-Uchida et al., 2012; Faget et al., 2016). More recent analysis combining optogenetics and electrophysiology has identified direct inhibition of VTA DA and GABA neurons by the NAc (Edwards et al., 2017; Yang et al., 2018), with relatively greater inhibition of VTA DA neurons in the mNAc circuit (Yang et al., 2018). Behaviorally, our findings are consistent with NAc suppression of feeding acting through these direct connections onto VTA DA neurons, as activation of VTA GABAergic neurons has been shown to inhibit food consumption and drive aversion (Tan et al., 2012; van Zessen et al., 2012). However, the relative contribution of both local and projecting GABAergic neurons in the VTA to feeding is not clear.

The research presented here contributes to an emerging body of evidence indicating inhibitory feedback in the mesolimbic and basal ganglia circuits, and its role in feeding and reward. Activation of NAc projections to LH GABAergic neurons has been shown to inhibit feeding (O'Connor et al., 2015). These LH GABAergic neurons induce feeding and reward behavior, in part mediated by projections to the VTA (Jennings et al., 2015; Nieh 
et al., 2015, 2016), leading to indirect control of VTA dopamine function and reward behavior by the NAc. The mNAc $\rightarrow$ VTA circuit described here bypasses the LH to directly influence feeding behavior. It is likely that this direct feedback to the midbrain not only influences food intake and drug seeking, but all behaviors that are regulated by mesolimbic function.

\section{References}

Anderson J, Konz E, Frederich R, Wood C (2001) Long-term weight-loss maintenance: a meta-analysis of US studies. Am J Clin Nutr 74:579-584.

Aronoff R, Matyas F, Mateo C, Ciron C, Schneider B, Petersen CC (2010) Long-range connectivity of mouse primary somatosensory barrel cortex. Eur J Neurosci 31:2221-2233.

Baldo BA, Kelley AE (2007) Discrete neurochemical coding of distinguishable motivational processes: insights from nucleus accumbens control of feeding. Psychopharmacology (Berl) 191:439-459.

Basso A, Kelley A (1999) Feeding induced by GABA(A) receptor stimulation within the nucleus accumbens shell: regional mapping and characterization of macronutrient and taste preference. Behav Neurosci 113:324-336.

Bocklisch C, Pascoli V, Wong JC, House DR, Yvon C, de Roo M, Tan KR, Lüscher C (2013) Cocaine disinhibits dopamine neurons by potentiation of GABA transmission in the ventral tegmental area. Science 341:15211525.

Edwards NJ, Tejeda HA, Pignatelli M, Zhang S, McDevitt RA, Wu J, Bass CE, Bettler B, Morales M, Bonci A (2017) Circuit specificity in the inhibitory architecture of the VTA regulates cocaine-induced behavior. Nat Neurosci 20:438-448.

Faget L, Osakada F, Duan J, Ressler R, Johnson AB, Proudfoot JA, Yoo JH, Callaway EM, Hnasko TS (2016) Afferent inputs to neurotransmitterdefined cell types in the ventral tegmental area. Cell Rep 15:2796-2808.

Gibson GD, Prasad AA, Jean-Richard-Dit-Bressel P, Yau JO, Millan E, Liu Y, Campbell EJ, Lim J, Marchant NJ, Power JM, Killcross S, Lawrence AJ, McNally GP (2018) Distinct accumbens shell output pathways promote versus prevent relapse to alcohol seeking. Neuron 98:512-520.

Hommel JD, Sears R, Georgescu D, Simmons DL, DiLeone RJ (2003) Local gene knockdown in the brain using viral-mediated RNA interference. Nat Med 9:1539-1544.

Jennings JH, Ung RL, Resendez SL, Stamatakis A, Taylor JG, Huang J, Veleta K, Kantak PA, Aita M, Shilling-Scrivo K, Ramakrishnan C, Deisseroth K, Otte S, Stuber GD (2015) Visualizing hypothalamic network dynamics for appetitive and consummatory behaviors. Cell 160:516-527.

Kelley AE, Baldo BA, Pratt WE, Will MJ (2005) Corticostriatal-hypothalamic circuitry and food motivation: integration of energy, action and reward. Physiol Behav 86:773-795.

Krause M, German P, Taha SA, Fields HL (2010) A pause in nucleus accumbens neuron firing is required to initiate and maintain feeding. J Neurosci 30:4746-4756.

Land BB, Narayanan NS, Liu RJ, Gianessi CA, Brayton CE, Grimaldi DM, Sarhan M, Guarnieri DJ, Deisseroth K, Aghajanian GK, DiLeone RJ (2014) Medial prefrontal D1 dopamine neurons control food intake. Nat Neurosci 17:248-253.

Lerner TN, Shilyansky C, Davidson TJ, Evans KE, Beier KT, Zalocusky KA, Crow AK, Malenka RC, Luo L, Tomer R, Deisseroth K (2015) Intactbrain analyses reveal distinct information carried by SNc dopamine subcircuits. Cell 162:635-647.

London T, Licholai J, Szczot I, Ali M, LeBlanc K, Fobbs W, Kravitz A (2018) Coordinated ramping of dorsal striatal pathways preceding food approach and consumption. J Neurosci 38:3547-3558.

Löw K, Aebischer P, Schneider BL (2013) Direct and retrograde transduction of nigral neurons with AAV6, 8, and 9 and intraneuronal persistence of viral particles. Hum Gene Ther 24:613-629.

Lu X, Ghasemzadeh M, Kalivas P (1998) Expression of D1 receptor, D2 receptor, substance $\mathrm{P}$ and enkephalin messenger RNAs in the neurons projecting from the nucleus accumbens. Neuroscience 82:767-780.

Maldonado-Irizarry C, Swanson C, Kelley A (1995) Glutamate receptors in the nucleus accumbens shell control feeding behavior via the lateral hypothalamus. J Neurosci 15:6779-6788.

Micha R, Peñalvo JL, Cudhea F, Imamura F, Rehm CD, Mozaffarian D (2017) Association between dietary factors and mortality from heart disease, stroke, and type 2 diabetes in the United States. JAMA 317:912-924.
Miner P, Borkuhova Y, Shimonova L, Khaimov A, Bodnar RJ (2010) GABA$A$ and GABA-B receptors mediate feeding elicited by the GABA-B agonist baclofen in the ventral tegmental area and nucleus accumbens shell in rats: reciprocal and regional interactions. Brain Res 1355:86-96.

Narayanan NS, Land BB, Solder JE, Deisseroth K, DiLeone RJ (2012) Prefrontal D1 dopamine signaling is required for temporal control. Proc Natl Acad Sci USA 109:20726-20731.

Nieh EH, Matthews GA, Allsop SA, Presbrey KN, Leppla CA, Wichmann R, Neve R, Wildes CP, Tye KM (2015) Decoding neural circuits that control compulsive sucrose seeking. Cell 160:528-541.

Nieh EH, Weele CM, Matthews GA, Presbrey KN, Wichmann R, Leppla CA, Izadmehr EM, Tye KM (2016) Inhibitory input from the lateral hypothalamus to the ventral tegmental area disinhibits dopamine neurons and promotes behavioral activation. Neuron 90:1286-1298.

O'Connor EC, Kremer Y, Lefort S, Harada M, Pascoli V, Rohner C, Lüscher C (2015) Accumbal D1R neurons projecting to lateral hypothalamus authorize feeding. Neuron 88:553-564.

Prado L, Luis-Islas J, Sandoval OI, Puron L, Gil MM, Luna A, Arias-García MA, Galarraga E, Simon SA, Gutierrez R (2016) Activation of glutamatergic fibers in the anterior NAc shell modulates reward activity in the aNAcSh, the lateral hypothalamus, and medial prefrontal cortex and transiently stops feeding. J Neurosci 36:12511-12529.

Pulman KG, Somerville E, Clifton PG (2012) Intra-accumbens baclofen, but not muscimol, increases second order instrumental responding for food reward in rats. PLoS One 7:e40057.

Reynolds S, Berridge K (2001) Fear and feeding in the nucleus accumbens shell: rostrocaudal segregation of GABA-elicited defensive behavior versus eating behavior. J Neurosci 21:3261-3270.

Richardson N, Roberts D (1996) Progressive ratio schedules in drug selfadministration studies in rats: a method to evaluate reinforcing efficacy. J Neurosci Methods 66:1-11.

Roitman MF, Wheeler RA, Carelli RM (2005) Nucleus accumbens neurons are innately tuned for rewarding and aversive taste stimuli, encode their predictors, and are linked to motor output. Neuron 45:587-597.

Salegio E, Samaranch L, Kells A, Mittermeyer G, Sebastian SW, Zhou S, Beyer J, Forsayeth J, Bankiewicz K (2013) Axonal transport of adenoassociated viral vectors is serotype-dependent. Gene Ther 20:348-352.

Snook KR, Hansen AR, Duke CH, Finch KC, Hackney AA, Zhang J (2017) Change in percentages of adults with overweight or obesity trying to lose weight, 1988-2014. JAMA 317:971-973.

Stratford T, Kelley A (1997) GABA in the nucleus accumbens shell participates in the central regulation of feeding behavior. J Neurosci 17:44344440.

Stratford T, Kelley A (1999) Evidence of a functional relationship between the nucleus accumbens shell and lateral hypothalamus subserving the control of feeding behavior. J Neurosci 19:11040-11048.

Stratford TR, Wirtshafter D (2012a) Evidence that the nucleus accumbens shell, ventral pallidum, and lateral hypothalamus are components of a lateralized feeding circuit. Behav Brain Res 226:548-554.

Stratford TR, Wirtshafter D (2012b) Effects of muscimol, amphetamine, and DAMGO injected into the nucleus accumbens shell on food-reinforced lever pressing by undeprived rats. Pharmacol Biochem Behav 101:499503.

Taha SA, Fields HL (2005) Encoding of palatability and appetitive behaviors by distinct neuronal populations in the nucleus accumbens. J Neurosci 25:1193-1202.

Taha SA, Fields HL (2006) Inhibitions of nucleus accumbens neurons encode a gating signal for reward-directed behavior. J Neurosci 26:217-222.

Tan KR, Yvon C, Turiault M, Mirzabekov JJ, Doehner J, Labouèbe G, Deisseroth K, Tye KM, Lüscher C (2012) GABA neurons of the VTA drive conditioned place aversion. Neuron 73:1173-1183.

Tervo D, Hwang BY, Viswanathan S, Gaj T, Lavzin M, Ritola KD, Lindo S, Michael S, Kuleshova E, Ojala D, Huang CC, Gerfen CR, Schiller J, Dudman JT, Hantman AW, Looger LL, Schaffer DV, Karpova AY (2016) A designer AAV variant permits efficient retrograde access to projection neurons. Neuron 92:372-382.

Urstadt KR, Kally P, Zaidi SF, Stanley B (2013) Ipsilateral feeding-specific circuits between the nucleus accumbens shell and the lateral hypothalamus: regulation by glutamate and GABA receptor subtypes. Neuropharmacology 67:176-182. 
U.S. Burden of Disease Collaborators (2018) The state of US health, 19902016: burden of diseases, injuries, and risk factors among US states. JAMA 319:1444-1472.

Usuda I, Tanaka K, Chiba T (1998) Efferent projections of the nucleus accumbens in the rat with special reference to subdivision of the nucleus: biotinylated dextran amine study. Brain Res 797:73-93.

van Zessen R, Phillips JL, Budygin EA, Stuber GD (2012) Activation of VTA GABA neurons disrupts reward consumption. Neuron 73:1184-1194.

Watabe-Uchida M, Zhu L, Ogawa SK, Vamanrao A, Uchida N (2012) Whole-brain mapping of direct inputs to midbrain dopamine neurons. Neuron 74:858-873.

Will M, Franzblau E, Kelley A (2003) Nucleus accumbens mu-opioids regulate intake of a high-fat diet via activation of a distributed brain network. J Neurosci 23:2882-2888.

Xia Y, Driscoll JR, Wilbrecht L, Margolis EB, Fields HL, Hjelmstad GO (2011) Nucleus accumbens medium spiny neurons target non- dopaminergic neurons in the ventral tegmental area. J Neurosci 31:7811-7816.

Yang H, de Jong JW, Tak Y, Peck J, Bateup HS, Lammel S (2018) Nucleus accumbens subnuclei regulate motivated behavior via direct inhibition and disinhibition of VTA dopamine subpopulations. Neuron 97:434449.e4.

Zahm D, Heimer L (1993) Specificity in the efferent projections of the nucleus accumbens in the rat: comparison of the rostral pole projection patterns with those of the core and shell. J Comp Neurol 327:220-232.

Zhang M, Balmadrid C, Kelley AE (2003) Nucleus accumbens opioid, GABaergic, and dopaminergic modulation of palatable food motivation: contrasting effects revealed by a progressive ratio study in the rat. Behav Neurosci 117:202-211.

Zhang M, Kelley A (2000) Enhanced intake of high-fat food following striatal mu-opioid stimulation: microinjection mapping and fos expression. Neuroscience 99:267-277. 